\title{
The potential of recombinant vesicular stomatitis virus-mediated virotherapy against metastatic colon cancer
}

\author{
MINORU YAMAKI $^{1}$, KATSUNORI SHINOZAKI ${ }^{1,6}$, TAKEMASA SAKAGUCHI $^{2}$, MARCIA MESECK $^{3}$, \\ OLIVER EBERT ${ }^{4}$, HIDEKI OHDAN ${ }^{5}$ and SAVIO L.C. WOO ${ }^{3}$
}

\author{
${ }^{1}$ Department of Surgery, Division of Frontier Medical Sciences, Programs for Biomedical Research, Graduate School of \\ Biomedical Sciences, ${ }^{2}$ Department of Virology, Basic Life Sciences, Institute of Biomedical and Health Sciences, \\ Hiroshima University, Hiroshima 734-8551, Japan; ${ }^{3}$ Department of Gene and Cell Medicine, Mount Sinai School of Medicine, \\ New York, NY 10029, USA; ${ }^{4}$ Second Medical Department, Klinikum Rechts der Isar, Technical University of Munich, \\ Munich D-81675, Germany; ${ }^{5}$ Department of Gastroenterological and Transplant Surgery, Applied Life Sciences, \\ Institute of Biomedical and Health Sciences, Hiroshima University, Hiroshima 734-8551, Japan
}

Received September 3, 2012; Accepted October 30, 2012

DOI: $10.3892 /$ ijmm.2012.1205

\begin{abstract}
Colorectal cancer (CRC) is the fourth most frequently diagnosed cancer and the second leading cause of cancer-related mortality in the United States. The liver and lung are the most common sites of distant metastasis of CRC. The approval of newer chemotherapeutic agents such as oxaliplatin, irinotecan, bevacizumab, cetuximab and panitumumab has significantly improved survival, yet the majority of patients still succumb to the disease in less than 2 years. Novel therapeutic agents that can provide significant clinical benefit for metastatic CRC patients are needed. Oncolytic vesicular stomatitis virus (VSV) is a promising tool as a cancer therapeutic agent. In this study, we examined the feasibility of repeated intravenous infusions of rVSV in multiple CRC lung metastases, compared with repeated hepatic arterial administration in multifocal CRC liver metastasis in immune competent rats. We established a multifocal liver metastases model or the multiple lung metastases model using a CRC cell line, $\mathrm{RCN}-\mathrm{H} 4$, implanted into syngeneic F344/DuCrj rats. 4.0x $10^{6}$ plaque-forming units (pfu) of recombinant VSV vectors expressing mutant (L289A) Newcastle disease virus fusion protein [rVSV-NDV/F(L289A)] were administered 3 times for 3 consecutive days locally via the hepatic artery for liver metastases or systemically via the penial vein for lung metastases. In the liver metastasis model, significantly enhanced survival was observed with rVSV$\mathrm{NDV} / \mathrm{F}(\mathrm{L} 289 \mathrm{~A})$-treated rats $(\mathrm{P}=0.0196)$. Median survival was 110 and 25 days, respectively. In addition, 4 out of 7 of
\end{abstract}

Correspondence to: Dr Katsunori Shinozaki, ${ }^{6}$ Present address: Department of Clinical Oncology, Hiroshima Prefectural Hospital, 1-5-54 Minamiku Ujinakanda, Hiroshima 734-8530, Japan

E-mail:k-shinozaki@hph.pref.hiroshima.jp

Key words: oncolytic vesicular stomatitis virus, metastatic colorectal cancer, virotherapy the rVSV-NDV/F(L289A)-treated rats demonstrated longterm survival exceeding 100 days. The long-term surviving rats were sacrificed to evaluate for residual malignancy. Liver tumors were not detected. In the lung metastasis model, median survival was 10 [VSV-NDV/F(L289A)-treated rats] and 7 days (control). Although survival was significantly prolonged $(\mathrm{P}<0.001)$, none of the rats achieved long-term survival. VSV virotherapy has potential for CRC liver and lung metastases, although systemic venous delivery is much less effective than locoregional delivery such as hepatic arterial infusion.

\section{Introduction}

Colorectal cancer (CRC) is the fourth most frequently diagnosed cancer and the second leading cause of cancer-related mortality in the United States. According to cancer statistics in 2012, 143,460 new cases of CRC are diagnosed with colon or rectal cancer, and 51,690 succumb to CRC, most with metastatic tumors (1). Approximately $50 \%$ of patients diagnosed with CRC will develop CRC liver metastases, and 70-80\% of these have unresectable metastatic liver disease (2). The lung is the second most frequent site of metastasis of CRC. Approximately $8-10 \%$ of patients develop pulmonary metastases after resection of the primary CRC (3). The majority of patients with metastatic CRC cannot be cured, although a subset of patients with liver and/or lung-isolated metastatic disease, local recurrence, or limited intra-abdominal disease is potentially curable with surgery. For other patients with metastatic CRC, treatment is palliative and generally consists of systemic chemotherapy. Significant progress in the systemic treatment of CRC has been achieved over the past twelve years, with various active drugs, either in combination or as single agents: 5-FU/LV, capecitabine, irinotecan, oxaliplatin, bevacizumab, cetuximab and panitumumab (4). Median overall survival for patients with metastatic CRC has increased from less than 9 months with no treatment to approximately 24 months. However, the majority of patients can not be cured of CRC, and succumb to the disease in less than 2 years. Novel 
therapeutic agents that can provide significant clinical benefit for patients with advanced CRC are urgently required.

Novel molecular therapies in pre-clinical animal models and early clinical trials to treat hepatic metastasis from CRC have been reported (5-7). Vesicular stomatitis virus (VSV) is a negative-strand RNA virus of the family Rhabdoviridae with potent oncolytic properties that is exquisitely sensitive to the antiviral actions of type 1 interferons (IFN- $\alpha / \beta)$ in normal but not in cancer cells $(8,9)$. It is considered to be due to the fact that IFN-responsive anti-viral pathways are defective in many types of tumors (8-11). We previously demonstrated that repeated administrations (every other day for 4 days, 3 injections total) of VSV-NDV/F(L289A), which is an engineered VSV expressing a fusogenic membrane glycoprotein from a heterologous virus, through a permanent cannula surgically implanted into the hepatic artery, led to sustained tumor-selective virus replication and substantially enhanced its oncolytic potential in the treatment of advanced multifocal hepatocellular carcinoma in the liver of rats (12).

In this study, we generated an orthotropic model of CRC in the livers or the lungs of syngeneic and immune competent rats and investigated the feasibility of repeated intravenous infusions of rVSV-NDV/F(L289A) for multiple CRC lung metastases, compared with the feasibility of repeated hepatic arterial infusions for multifocal CRC liver metastases in immune competent rats.

\section{Materials and methods}

Cell lines and culture conditions. The rat CRC cell line RCN-H4 was obtained from RIKEN BioResource Center Cell Bank (Ibaraki, Japan). RCN-H4 is a subclone established by Inoue et al (13) according to Fidler's method; it has a high potency for forming experimental liver metastatic tumors. RCN-H4 was maintained in RPMI-1640 medium (SigmaAldrich, St. Louis, MO, USA) containing 10\% heat-inactivated fetal bovine serum (FBS) and $0.05 \%$ penicillin-streptomycin solution (were from Sigma-Aldrich).

Recombinant VSV-NDV/F(L289A) vectors. Recombinant VSV vector expressing mutant (L289A) Newcastle disease virus fusion protein [rVSV-NDV/F(L289A)] has previously been described (14). Viral titers of working stocks were determined on BHK-21 cells by using standard plaque assays. A resulting titer for $\mathrm{rVSV}-\mathrm{NDV} / \mathrm{F}(\mathrm{L} 289 \mathrm{~A})$ was $1.4 \times 10^{9}$ plaque-forming units $(\mathrm{pfu}) / \mathrm{ml}$.

In vitro cytotoxicity assay. $\mathrm{RCN}-\mathrm{H} 4$ cells were seeded in 24-well plates at $5 \times 10^{4}$ cells/well with RPMI-1640 medium containing $10 \%$ FBS. After two days, they were infected with rVSV-NDV/F(L289A) at a multiplicity of infection (MOI) of $0.0001,0.001,0.01$ or 0.1 . Cell viability was measured at the indicated time points after infection by the 3-(4,5-dimethylthiazol-2-yl)-2,5-diphenyltetrazolium bromide (MTT) assay (Cell Proliferation kit I; Roche Diagnostics, Indianapolis, IN, USA). All cell viability results are expressed as percentage of viable cells compared to mock-infected control at each time point. To test the effect of fresh serum on rVSV tumor cell killing, RCN-H4 was cultured in RPMI-1640 medium containing $10 \%$ heat-inactivated rat serum or fresh serum. Rat serum was collected from F344/DuCrj rats. Fresh or heat-inactivated serum was diluted with RPMI-1640 medium (1:9 dilution).

Orthotopically multifocal CRC metastasis model in syngeneic rats. Inbred male $\mathrm{F} 344 / \mathrm{DuCrj}$ rats (7-8 weeks old; 150-180 g) were purchased from Charles River Japan, Inc. (Chiba, Japan) and housed in a specific pathogen-free environment under standard conditions. All procedures involving animals were approved by the Hiroshima University Animal Ethics Committee and were performed according to their guidelines. Rats were anesthetized with $100 \mathrm{mg} / \mathrm{kg}$ ketamine intraperitoneally and isoflurane using an inhalation anesthesia system (Bio Machinery, Chiba, Japan). Subsequently, rats were implanted with $5 \times 10^{6}$ syngeneic RCN-H4 cells in $20 \mu \mathrm{l}$ of RPMI-1640 in the left lateral and right central lobe of the liver to establish multiple liver metastases of CRC. In order to establish multiple lung metastases of CRC, rats were infused with $1 \times 10^{6}$ syngeneic RCN-H4 cells in $1 \mathrm{ml}$ of RPMI-1640 via the penial vein.

Surgical placement of an indwelling intrahepatic artery cannula for repeated vector administration. Rats were anesthetized and underwent laparotomy 21 days after tumor cell implantation. The hepatic vessels (common hepatic artery, proper hepatic artery, and gastroduodenal artery) were dissected with the aid of an operating microscope. The Preclinical Mini-Port implantable access device (Deltec, Inc., St. Paul, MN, USA) was used to administer the vector repeatedly via the hepatic artery. After ligation of the gastroduodenal artery with 7-0 Prolene (Ethicon, Somerville, NJ, USA), a 2-French clear Polyurethane catheter (outer diameter $0.63 \mathrm{~mm}$, inner diameter $0.30 \mathrm{~mm}$ ) was inserted into the gastroduodenal artery. The common hepatic artery was ligated to prevent the rVSV vector from flowing backward. The Preclinical Mini-Port device was then implanted into a subcutaneous pocket in the umbilical area.

$r V S V-N D V / F(L 289 A)$ virotherapy. rVSV-NDV/F(L289A) vector $\left(4.0 \times 10^{6} \mathrm{pfu}\right)$ in $1 \mathrm{ml}$ of phosphate-buffered saline (PBS) was administered 3 times for 3 consecutive days via the port system. CRC lung metastasis models were infused intravenously via the penial vein at 14 days after tumor cell infusion.

Assessment of serum chemistries. Blood samples were collected from the inferior vena cava of each rat on Days 1 and 3. The levels of alanine transferase (ALT), aspartate transferase (AST), creatinine, and blood urea nitrogen (BUN) were determined at the Chemistry Laboratory at Fukuyama Medical Laboratories (Fukuyama, Japan).

Histology and immunohistochemical stainings. At indicated time points after rVSV-NDV/F(L289A) vector infusion into the hepatic artery, animals were sacrificed. Livers were fixed in $4 \%$ paraformaldehyde overnight, and were then paraffin-embedded. Sections $(5 \mu \mathrm{m})$ were subjected to either hematoxylin and eosin (H\&E) staining for histological analysis or immunohistochemistry using rabbit polyclonal antibodies against cleaved caspase-3 (Cell Signaling Technology, Danvers, MA, USA) for assessment of rVSV-mediated apoptosis. Immunohistochemistry sections were counterstained with hematoxylin. 
Statistical analysis. For comparison of the means of two groups, the two-sided Student's t-test for independent groups was applied to determine statistical significance. Survival curves of animals were plotted according to the Kaplan-Meier method. Statistical significance in different treatment groups was compared using the log-rank test. All statistical analyses were performed using StatView software version 5.0 (Abacus Concepts, Berkeley, CA).

\section{Results}

Oncolytic activities of $r V S V-N D V / F(L 289 A)$ in rat CRC cells in vitro. To assess the cytotoxic effect of rVSV-NDV/F(L289A) on the rat CRC cell line, we infected RCN-H4 cells with the virus at various MOIs. Percentage of cell survival was quantified by MTT assays as a fraction of mock-infected cells at each time point (Fig. 1). The rVSV-NDV/F(L289A) killed tumor cells in a dose-dependent manner up to $24 \mathrm{~h}$. At $32 \mathrm{~h}$, almost $100 \%$ of the cells were killed even at $0.0001 \mathrm{MOI}$ of rVSVNDV/F(L289A). This suggested that extremely low doses of rVSV-NDV/F(L289F) can kill RCN-H4 cells efficiently in vitro.

Animal models of multifocal CRC liver or multiple CRC lung metastasis. At 21 days after implantation of RCN-H4 cells in the left lateral and right central lobe of the liver, $100 \%$ of animals developed two sites of CRC lesions of up to $20 \mathrm{~mm}$ in diameter in their livers. Peritoneal dissemination was not detected. At 14 days following venous infusion of RCN-H4 cells, $100 \%$ of animals developed multiple CRC metastatic lesions of up to $2 \mathrm{~mm}$ in diameter in their lungs (data not shown).

Absence of organ toxicity after repeated hepatic arterial infusions or intravenous infusions of $r V S V-N D V / F(L 289 A)$. The maximum tolerated dose of rVSV-NDV/F(L289A) of immune competent rats was $1.3 \times 10^{7} \mathrm{pfu}(15)$. We evaluated the potential hepatotoxicity after three injections of $4.0 \times 10^{6} \mathrm{pfu}$ of rVSV-NDV/F(L289A) through the indwelling intrahepatic artery catheter. Rats with liver metastases were infused with rVSV-NDV/F(L289A) $(n=3)$ on Days 0-2, or sham operation was performed $(n=3)$. Blood samples were obtained from each rat on Days 1 and 3. The kinetic profiles of serum transaminase levels (aspartate aminotransferase and alanine aminotransferase) were determined (Fig. 2A). When rVSV$\mathrm{NDV} / \mathrm{F}(\mathrm{L} 289 \mathrm{~A})$ was administered via the hepatic artery, the common hepatic artery was ligated to prevent rVSV-NDV/ F(L289A) from flowing backward. Transient elevations of serum transaminases (AST and ALT) were seen at Day 1 after treatment in both groups, but the levels rapidly returned to baseline at Day 3. There were no significant differences in both groups. Additionally, the histology of the neighboring hepatic parenchyma was completely normal (Fig. 2B). Therefore, transient elevation of serum transaminase was possibly due to ligation of the common hepatic artery, and there was no remarkable hepatotoxicity associated with three injections of rVSV-NDV/F(L289A) via the hepatic artery.

In addition, we evaluated potential organ toxicity after 3 injections of intravenous infusions of $4.0 \times 10^{6} \mathrm{pfu}$ of rVSV$\mathrm{NDV} / \mathrm{F}(\mathrm{L} 289 \mathrm{~A})$ in the multiple lung metastases model. Blood samples were obtained from each rat on Days 1 and 3. The kinetic profiles of serum transaminases (AST and ALT),

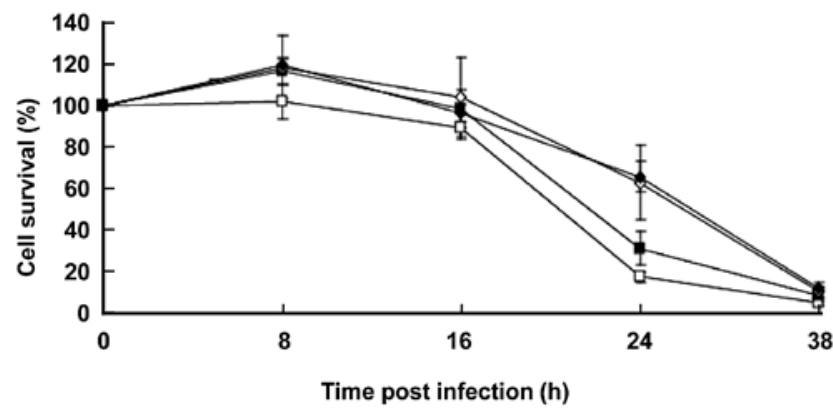

Figure 1. Oncolytic effect of recombinant VSV vector on a rat CRC cell line $\mathrm{RCN}-\mathrm{H} 4$ in vitro. RCN-H4 cells were infected with rVSV-NDV/F(L289A) at different MOIs. Cell viability was measured at the indicated time points using MTT assay. All cell viability results are expressed as percentage of viable cells compared to mock-infected control at each time point. Data are expressed as means \pm standard error of the mean of triplicate experiments. MOIs used for all experiments were as follows: solid diamonds, MOI 0.0001; open diamonds, MOI 0.001; solid squares, MOI 0.01; open squares, MOI 0.1. Experiments were carried out in triplicates.

creatinine and BUN as indications of liver and kidney damage, respectively, were determined (Fig. 3). There were no significant differences of AST, ALT, creatinine and BUN in both groups, indicating a lack of hepato- and nephrotoxicity associated with repeated intravenous infusions of rVSVNDV/F(L289A).

In vivo antitumor efficacy of repeated hepatic arterial infusions in multifocal CRC liver metastases. To demonstrate the antitumor efficacy of repeated hepatic arterial infusions of rVSV-NDV/F(L289A), rats were sacrificed at indicated time points (Days 0, 1, 3 and 7) and the tumor-bearing livers were excised for histological analysis. In the control liver sections, most areas within the tumor were viable tumor cells (Fig. 4A). By contrast, we detected large necrotic areas within the tumor in the rVSV-NDV/F(L289A)-treated liver at Day 7 (Fig. 4D). We measured the percentage of necrotic areas to the total area in representative tumor sections by using a BZ-H1M3 program and image analyzer (Keyence, Osaka) (Fig. 4E). The percentage of necrotic areas before treatment was $11.5 \%$. At Day 1, after a single infusion of rVSV-NDV/F(L289A), the percentage was $31.4 \%$ (Fig. 4B). At Day 3, after repeated infusions of rVSV-NDV/F(L289A), the percentage was $83.2 \%$ (Fig. 4C). Finally, the percentage of necrotic areas further increased to $92.3 \%$ at Day 7 (Fig. 4D). Liver parenchyma neighboring tumors showed no signs of pathology. These results suggested that 3 injections of $\mathrm{rVSV}-\mathrm{NDV} / \mathrm{F}(\mathrm{L} 289 \mathrm{~A})$ through the hepatic artery resulted in tumor-selective necrosis and no remarkable hepatotoxicity.

In order to elucidate the mechanism of the antitumor effect by rVSV, we analyzed tumor samples obtained at Day 0 (before treatment), Days 1 and 3 by immunohistochemistry using rabbit polyclonal antibodies against activated caspase-3. Caspase-3 is one of the key executioners of apoptosis, as it is either partially or totally responsible for the proteolytic cleavage of many key proteins such as the nuclear enzyme polymerase (16). rVSV causes lysis of infected tumor cells through activation of apoptotic pathways (17). At Day 0, minimal spontaneous necrotic areas within the tumor lesions were found (Fig. 5A), which were stained indistinctly 
A $\square$ PBS

- rVSV-NDVIF(L289A)

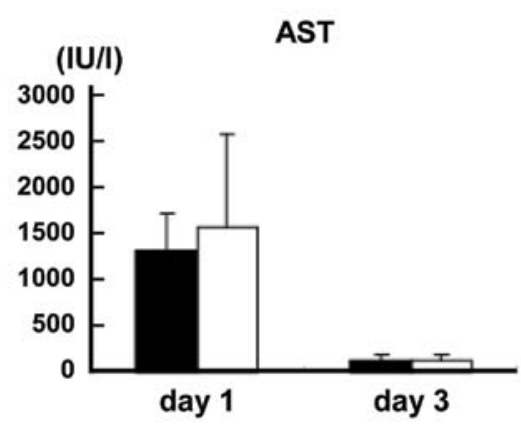

B

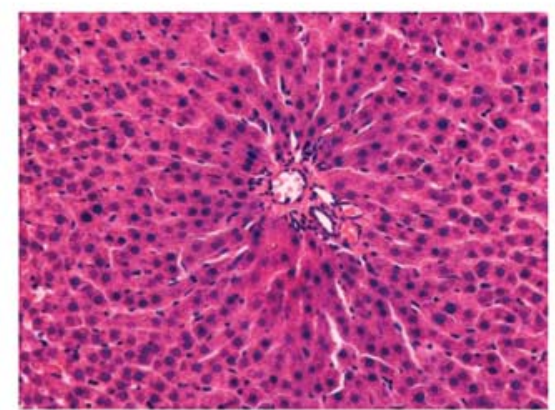

1 day after treatment
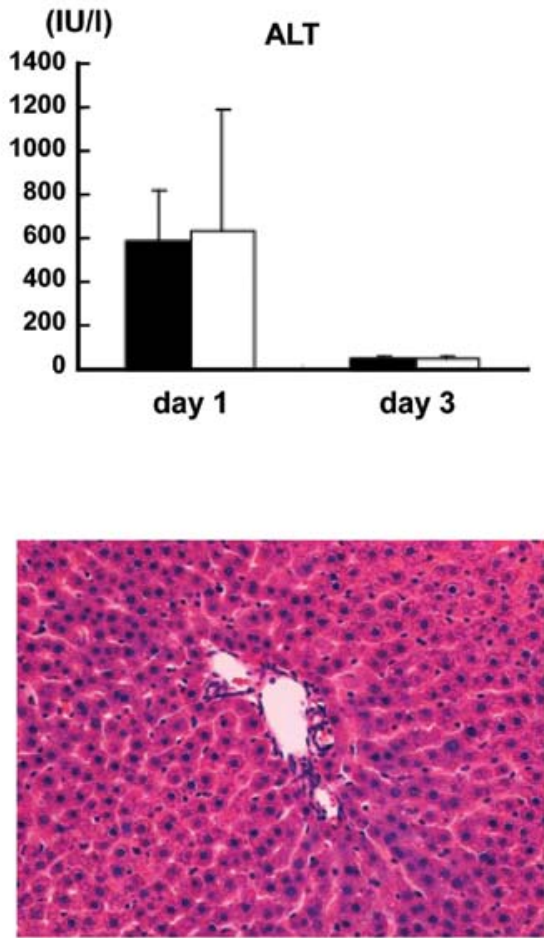

3 days after treatment

Figure 2. Absence of hepatotoxicity after repeated hepatic arterial infusions of VSV. (A) Blood samples were collected at indicated time points from sets of tumor-bearing rats with repeated hepatic arterial infusions of rVSV-NDV/F(L289A) at 3 times $(n=3)$ or from sham-operated rats ( $n=3)$. Serum transaminase [aspartate aminotransferase (AST) and alanine aminotransferase (ALT)] levels were determined. Bars represent mean values and error bars represent standard errors. (B) Representative H\&E stained sections of the liver obtained at Days 1 and 3 from tumor-bearing rats after repeated hepatic arterial infusion of rVSVNDV/F(L289A) (original magnification, x20).

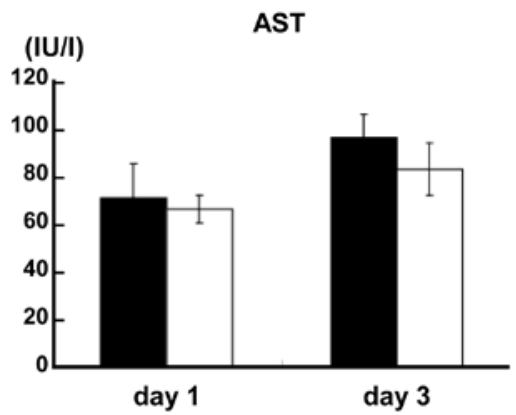

Creatinine

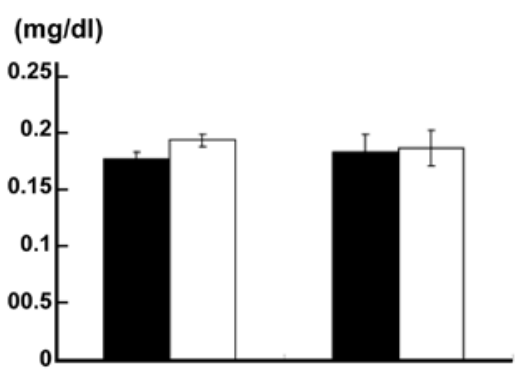

day 1

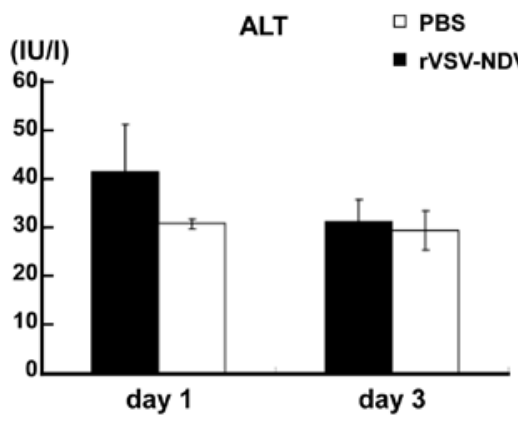

BUN

(mg/dl)

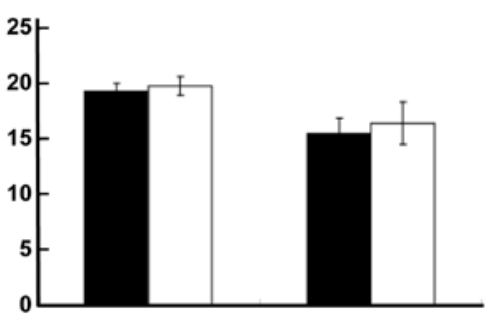

day 1 day 3

Figure 3. Absence of hepatotoxicity and nephrotoxicity after repeated intravenous infusions of VSV. Blood samples were collected at indicated time points from sets of tumor-bearing rats with 3 injections of intravenous infusion of rVSV-NDV/F(L289A) (n=3) or PBS ( $=3$ ). Serum transaminase [aspartate aminotransferase (AST) and alanine aminotransferase (ALT)], creatinine, and BUN levels were determined. Bars represent mean values and error bars represent standard error from three independent experiments. 
A

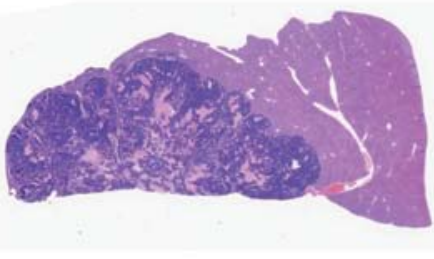

C
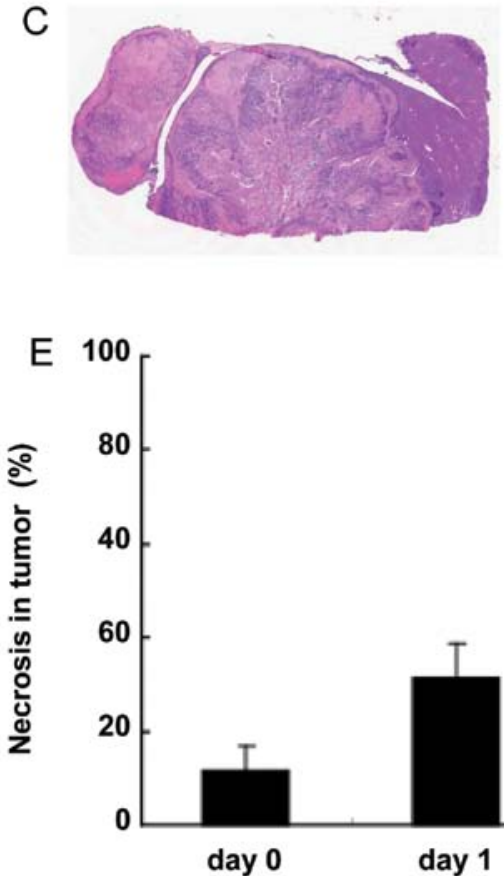

day 0

day 1
B

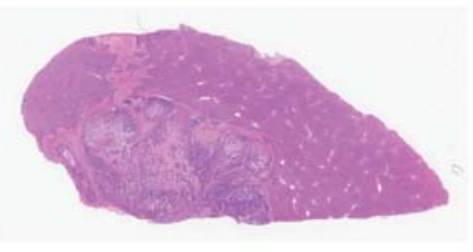

D

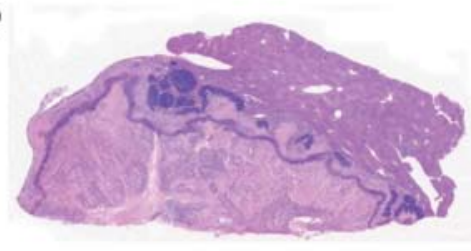

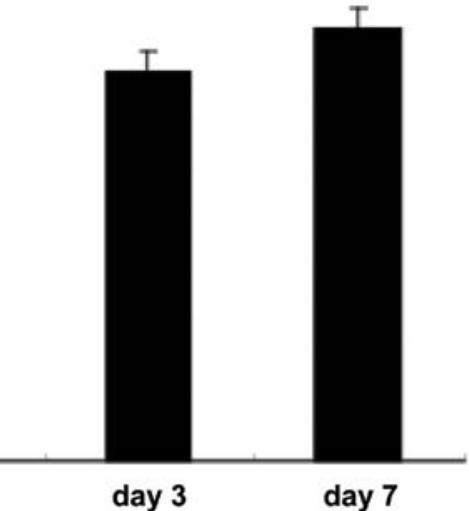

Figure 4. Response of tumor in rats treated with rVSV-NDV/F(L289A). (A-D) Representative H\&E stained sections of the liver obtained from tumor-bearing rats at (A) Day 0 (before treatment), (B) Day 1, (C) Day 3, and (D) Day 7, (original magnification, x1). (E) Necrosis in tumor was quantified by using a BZ-H1M3 program and image analyzer at indicated time points. Data are shown as means \pm standard deviation.

by cleaved caspase- 3 antibody (Fig. 5B). By contrast, at Day 1, we detected more necrotic areas in the tumor lesions (Fig. 5C), which were stained diffusely positive by cleaved caspase-3 antibody (Fig. 5D). At Day 3, most areas within the tumors were necrotic (Fig. 5E). A few viable tumor cells were detected at the rim of the tumor, which were stained weakly (Fig. 5F). It was suggested that rVSV-NDV/F(L289A) induced the apoptosis of tumor cells.

Prolonged survival of CRC liver metastasis-bearing rats treated with hepatic arterial infusions of $r V S V-N D V /$ $F(L 289 A)$. To assess the potential of rVSV-NDV/F(L289A) as a therapeutic agent for multifocal CRC liver metastasis, rats bearing two sites of CRC tumors in their livers with sizes $\leq 20 \mathrm{~mm}$ in diameter were randomly assigned to 3 injections of hepatic arterial infusions with $4.0 \times 10^{6} \mathrm{pfu}$ of rVSV-NDV/F(L289A) $(n=7)$ or subjected to sham operation $(n=8)$ and survival was followed (Fig. 6). Sham-operated rats started to die of tumor progression in 11 more days and all of them expired at 68 days (median survival, 25 days). Autopsy revealed massive liver metastases. By contrast, rats treated with intrahepatic arterial infusion of rVSV showed significantly prolonged survival $(\mathrm{P}=0.0196)$. By 100 days post-treatment, the median survival had not been reached.
Autopsy of the VSV-treated rats that died demonstrated multiple lung metastases and solitary liver metastases. However, we did not detect massive hepatomegaly with disseminated liver metastases nor peritoneal dissemination. The long-term surviving rats were sacrificed at 190 days after treatment and evaluated for residual malignancy. No metastatic liver tumors were detected, although some rats had metastatic lung tumors.

Prolonged survival of CRC lung metastasis-bearing rats treated with intravenous infusions of rVSV-NDV/F(L289A). To assess the potential of rVSV-NDV/F(L289A) as a therapeutic agent for multiple CRC lung metastases, rats bearing multiple lung metastases were randomly assigned to 3 injections of intravenous infusions with $4.0 \times 10^{6} \mathrm{pfu}$ of rVSV-NDV/F(L289A) $(n=13)$ or PBS $(n=15)$ via the penial vein, and survival was followed (Fig. 7). PBS-treated rats started to die of tumor progression in 5 more days and all of them expired at 9 days (median survival, 7 days). The rVSVNDV/F(L289A)-treated rats survived until 28 days post vector injection (median survival, 10 days). Although the differential survival rates were statistically significant by log-rank test analysis $(\mathrm{P}<0.001)$, no rats treated with $\mathrm{rVSV}-\mathrm{NDV} / \mathrm{F}(\mathrm{L} 289 \mathrm{~A})$ achieved long-term survival. 
A

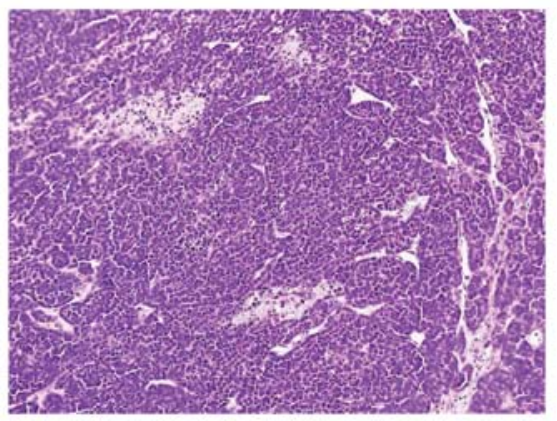

C

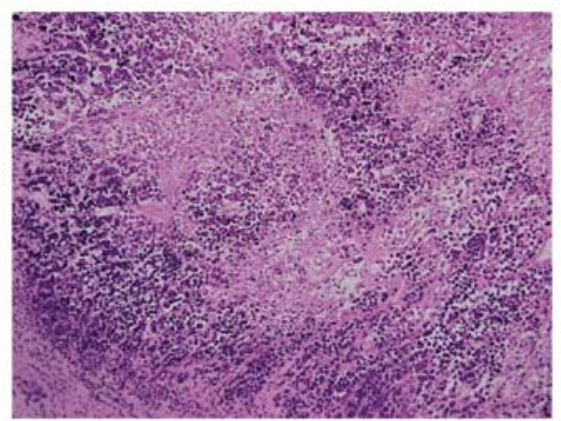

$\mathrm{E}$

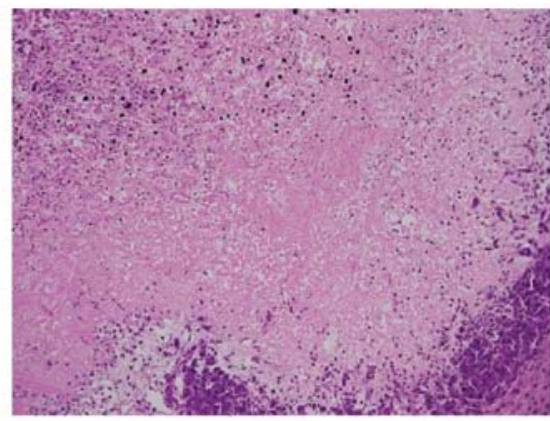

B

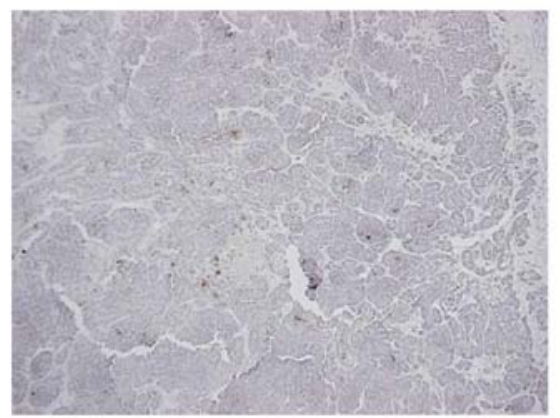

D

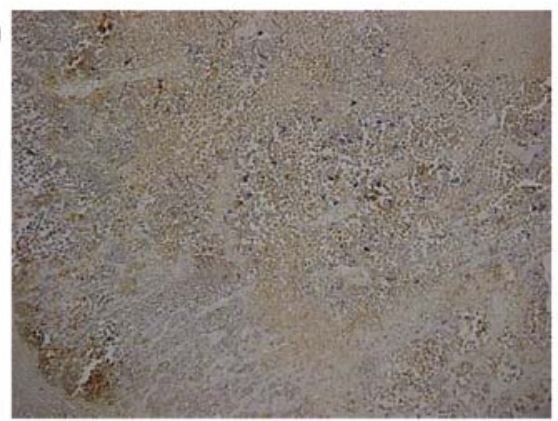

$\mathrm{F}$

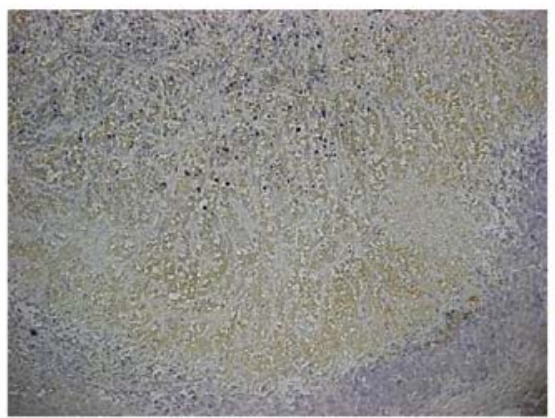

Figure 5. Virus-induced apoptosis in tumor tissues with repeated administration of VSV vector. (A, C and E) H\&E staining and (B, D and F) immunohistochemistry against cleaved caspase-3 (positive staining appears in brown) of representative tumor sections obtained at (A and B) Day 0 (before treatment), (C and D) Day 1 and (E and F) Day 3 (original magnification, x10).

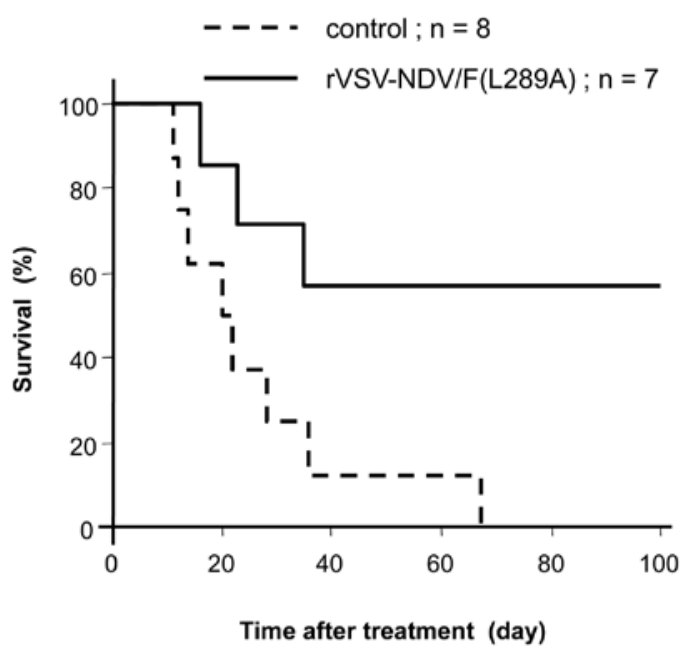

Figure 6. Kaplan-Meier survival curves of rats with multifocal CRC lesions in the liver after repeated hepatic arterial infusions of VSV vs. sham operation. Tumor-bearing rats were repeatedly infused with $4.0 \times 10^{6} \mathrm{pfu}$ of rVSV-NDV/F(L289A) via the hepatic artery (solid line) or underwent sham operation (dashed line) on Day 0 , and followed for survival. The survival advantage for VSV administered rats was statistically significant compared to control rats $(\mathrm{P}=0.0196)$. The results were combined from two consecutive sets of animals with stratification.
Effect of fresh rat serum on $r V S V-N D V / F(L 289 A)$-mediated tumor killing. This study suggested that antitumor efficacy of intravenous infusion of rVSV-NDV/F(L289A) against CRC lung metastasis was strongly reduced, compared with locoregional delivery such as hepatic arterial infusion against CRC liver metastasis. Locoregional delivery is generally applied with the intention of obtaining a high drug concentration within the tumor tissue and low systemic drug levels avoiding systemic toxicity. Intravenous infusion might reduce the local concentration of rVSV within the tumors. To assess the effect of rat serum on rVSV-NDV/F(L289A)-mediated tumor cell killing, tumor cells were infected with rVSV-NDV/F(L289A) at an MOI of 0.01 in RPMI-1640 medium containing $10 \%$ rat fresh serum or $10 \%$ rat heat-inactivated serum. rVSV-mediated oncolysis at $24 \mathrm{~h}$ after infection was significantly suppressed by rat fresh serum ( $\mathrm{P}=0.0015)$ (Fig. 8).

\section{Discussion}

Oncolytic virotherapy is expected to prove valuable for treating cancer patients. VSV as well as other viruses including adenovirus, herpes simplex virus, reovirus, autonomous parvovirus, Newcastle disease virus, measles virus and several more have 


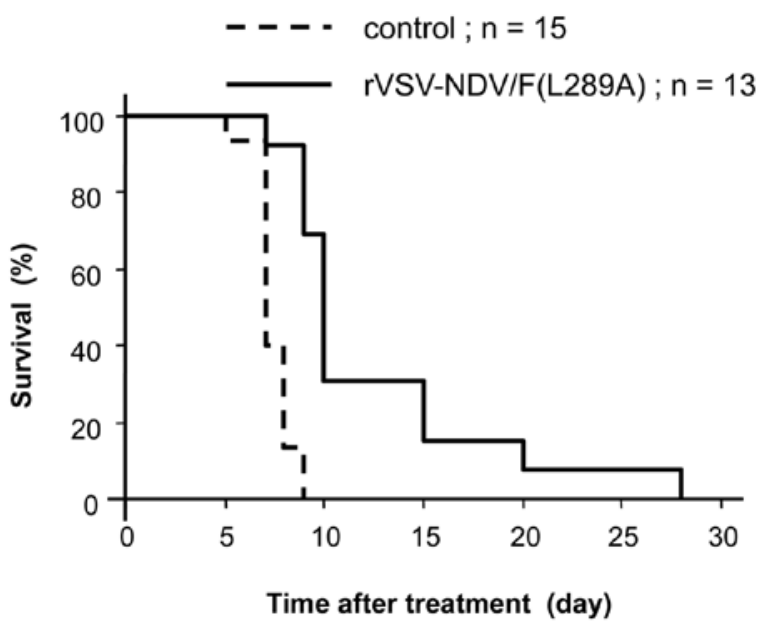

Figure 7. Kaplan-Meier survival curves of rats with multifocal CRC lesions in the lung after repeated intravenous infusions of VSV vs. PBS. Tumor-bearing rats were repeatedly infused with $4.0 \times 10^{6} \mathrm{pfu}$ of rVSV-NDV/F(L289A) via the penial vein (solid line) or infused with PBS (dashed line) on Day 0, and followed for survival. The survival advantage for VSV administered rats was statistically significant compared to control rats $(\mathrm{P}<0.001)$. The results were combined from two consecutive sets of animals with stratification.

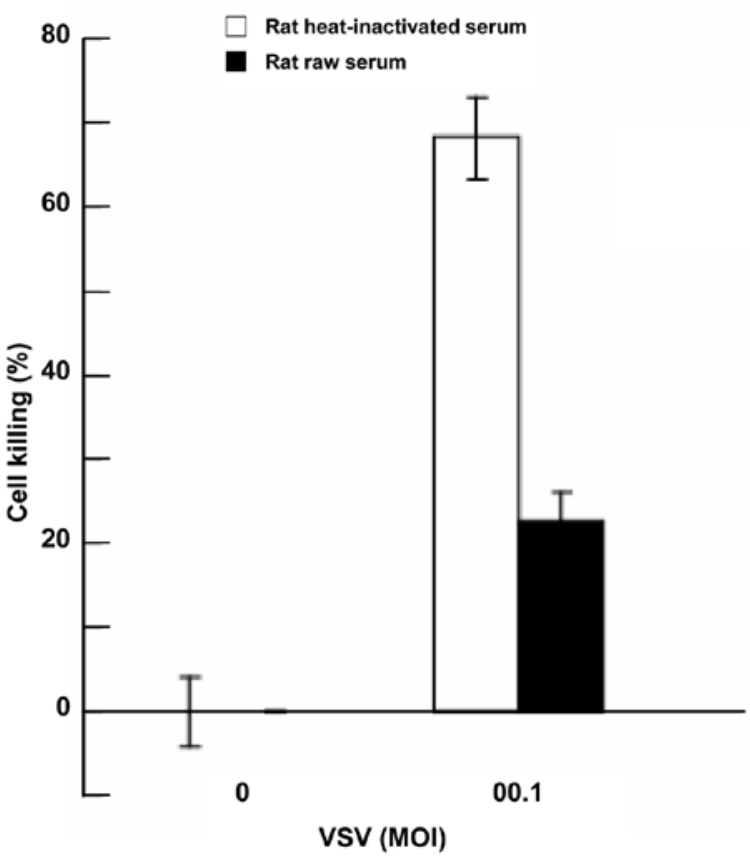

Figure 8. Effect of rat fresh serum on rVSV-NDV/F(L289A)-mediated tumor killing in vitro. $\mathrm{RCN}-\mathrm{H} 4$ cells were infected with $\mathrm{rVSV}-\mathrm{NDV} / \mathrm{F}(\mathrm{L} 289 \mathrm{~A})$ at an MOI of 0.01 and maintained in the RPMI-1640 medium containing $10 \%$ rat fresh serum or $10 \%$ rat heat-inactivated serum. Cell viability was measured at $24 \mathrm{~h}$ after infection using MTT assay. Data are expressed as means \pm standard errors from triplicate experiments.

been developed as oncolytic agents for cancer treatment (18). Compared with other replication-competent oncolytic vectors, VSV is very attractive as an oncolytic agent. The occurrence of antibodies against VSV in the general human population is extremely low, except in those regions where it is endemic, such as Georgia, USA, and Central America $(19,20)$. In addition, VSV can infect numerous types of tumor cells due to the widely tropic nature of the VSV G protein (21). Oncolytic virus therapy has the potential to destroy a tumor mass of unlimited size, if indeed productive virus infection spreads from one infected cell to another (22). However, the use of oncolytic viruses in destroying tumors in clinical trials has not yet been very successful in most cases (23). This indicates that the infectious virus dose can not spread efficiently through a tumor mass. In the present study, we demonstrated that repeated hepatic arterial infusions of rVSV-NDV/F(L289A) in multifocal liver metastases from CRC dramatically increased antitumor efficacy and caused long-term survival (more than 100 days) in the majority of the treated animals. We previously reported that a single hepatic arterial infusion of rVSV led to prolongation of rats bearing multiple CRC liver metastases without any long-term survivor (24). This difference in antitumor efficacy in vivo suggests that repeated administrations might cause rVSV to spread more efficiently within the tumor lesion. Repeated virus administration might increase tumor vessel leakage.

The lung is the second most frequent site of metastasis of CRC. To assess the potential of repeated infusions of rVSV in pulmonary CRC metastases, we established the multiple pulmonary metastases models with the same tumor cell line in syngeneic rats. To treat pulmonary CRC metastases with rVSV-NDV/F(L289A), the same dose of virus was used. The only difference was the injection route, which was via the penial vein. Although the differential survival rates were statistically significant by log-rank test analysis $(\mathrm{P}<0.001)$, no rats treated with rVSV-NDV/F(L289A) achieved longterm survival. When an oncolytic virus is given systemically to animals (for example, by intravenous injection), there are many barriers that prevent it from reaching the tumor and infecting cancer cells. The virus enters the circulation, where it can be quickly neutralized through absorption by blood cells, through the complement cascade or by neutralizing antibodies (25). We examined the effect of rat serum on rVSV-NDV/F(L289A)-mediated tumor cell killing. We confirmed that rVSV-mediated oncolysis could be impeded by an antiviral activity present in rat fresh serum. The mechanism of inactivation of rVSV by rat fresh serum might involve the components of the classical $\mathrm{C}$ pathway through $\mathrm{C} 3 \mathrm{~b}$ and a nonimmunoglobulin serum factor (26). To treat metastatic CRC more effectively with intravenous infusion of rVSV, we will need to overcome these barriers. Depending on a virus's clearance, a marked increase in the local concentration of the virus could be achieved by injection into the feeding artery.

In the present study, we demonstrated that repeated infusions of rVSV-NDV/F(L289A) were both effective and safe in the treatment of multifocal liver metastases as well as lung metastases of CRC in immune competent rat models, although systemic venous delivery is less effective than locoregional delivery such as hepatic arterial infusion. Locoregional deliver of rVSV could avoid its inactivation by fresh serum to maximize its antitumor efficacy.

\section{Acknowledgements}

The study was supported by Grants-in-Aid for Scientific Research (C) 16591322 from the Ministry of Education, Science, Sports, Culture and Technology of Japan. 


\section{References}

1. Siegel R, Naishadham D and Jemal A: Cancer statistics, 2012. CA Cancer J Clin 62: 10-29, 2012.

2. Wieser M, Sauerland S, Arnold D, Schmiegel W and ReinacherSchick A: Peri-operative chemotherapy for the treatment of resectable liver metastases from colorectal cancer: A systematic review and meta-analysis of randomized trials. BMC Cancer 10: 309, 2010.

3. Murata S, Moriya Y, Akasu T, Fujita S and Sugihara K: Resection of both hepatic and pulmonary metastases in patients with colorectal carcinoma. Cancer 83: 1086-1093, 1998.

4. Wolpin BM and Mayer RJ: Systemic treatment of colorectal cancer. Gastroenterology 134: 1296-1310, 2008.

5. Havlik R, Jiao LR, Nicholls J, Jensen SL and Habib NA: Gene therapy for liver metastases. Semin Oncol 29: 202-208, 2002

6. Mayer-Kuckuk P, Banerjee D, Kemeny N, Fong Y and Bertino JR: Molecular therapies for colorectal cancer metastatic to the liver. Mol Ther 5: 492-500, 2002.

7. Ruan DT and Warren RS: Liver-directed therapies in colorectal cancer. Semin Oncol 32: 85-94, 2005.

8. Stojdl DF, Lichty B, Knowles S, et al: Exploiting tumor-specific defects in the interferon pathway with a previously unknown oncolytic virus. Nat Med 6: 821-825, 2000.

9. Balachandran $\mathrm{S}$ and Barber GN: Vesicular stomatitis virus (VSV) therapy of tumors. IUBMB Life 50: 135-138, 2000.

10. Balachandran S, Porosnicu M and Barber GN: Oncolytic activity of vesicular stomatitis virus is effective against tumors exhibiting aberrant p53, Ras, or myc function and involves the induction of apoptosis. J Virol 75: 3474-3479, 2001.

11. Balachandran S and Barber GN: Defective translational control facilitates vesicular stomatitis virus oncolysis. Cancer Cell 5: 51-65, 2004.

12. Shinozaki K, Ebert O and Woo SL: Eradication of advanced hepatocellular carcinoma in rats via repeated hepatic arterial infusions of recombinant VSV. Hepatology 41: 196-203, 2005.

13. Inoue $Y$, Kashima Y, Aizawa $\mathrm{K}$ and Hatakeyama K: A new rat colon cancer cell line metastasizes spontaneously: biologic characteristics and chemotherapeutic response. Jpn J Cancer Res 82 90-97, 1991.
14. Ebert O, Shinozaki K, Kournioti C, Park MS, Garcia-Sastre A and Woo SL: Syncytia induction enhances the oncolytic potential of vesicular stomatitis virus in virotherapy for cancer. Cancer Res 64: 3265-3270, 2004

15. Shinozaki K, Ebert O, Kournioti C, Tai YS and Woo SL: Oncolysis of multifocal hepatocellular carcinoma in the rat liver by hepatic artery infusion of vesicular stomatitis virus. Mol Ther 9: 368-376, 2004

16. Fernandes-Alnemri T, Litwack G and Alnemri ES: CPP32, a novel human apoptotic protein with homology to Caenorhabditis elegans cell death protein Ced-3 and mammalian interleukin-1 beta-converting enzyme. J Biol Chem 269: 30761-30764, 1994.

17. Kopecky SA, Willingham MC and Lyles DS: Matrix protein and another viral component contribute to induction of apoptosis in cells infected with vesicular stomatitis virus. J Virol 75: 12169-12181, 2001.

18. Bell JC, Lichty B and Stojdl D: Getting oncolytic virus therapies off the ground. Cancer Cell 4: 7-11, 2003.

19. Giedlin MA, Cook DN and Dubensky TW Jr: Vesicular stomatitis virus: an exciting new therapeutic oncolytic virus candidate for cancer or just another chapter from Field's Virology? Cancer Cell 4: 241-243, 2003.

20. Lichty BD, Power AT, Stojdl DF and Bell JC: Vesicular stomatitis virus: re-inventing the bullet. Trends Mol Med 10: 210-216, 2004.

21. Barber GN: Vesicular stomatitis virus as an oncolytic vector Viral Immunol 17: 516-527, 2004.

22. McCormick F: Future prospects for oncolytic therapy. Oncogene 24: 7817-7819, 2005

23. Everts B and van der Poel HG: Replication-selective oncolytic viruses in the treatment of cancer. Cancer Gene Ther 12: 141-161, 2005.

24. Shinozaki K, Ebert O and Woo SL: Treatment of multi-focal colorectal carcinoma metastatic to the liver of immune-competent and syngeneic rats by hepatic artery infusion of oncolytic vesicular stomatitis virus. Int J Cancer 114: 659-664, 2005.

25. Parato KA, Senger D, Forsyth PA and Bell JC: Recent progress in the battle between oncolytic viruses and tumours. Nat Rev Cancer 5: 965-976, 2005.

26. Mills BJ and Cooper NR: Antibody-independent neutralization of vesicular stomatitis virus by human complement. I. Complement requirements. J Immunol 121: 1549-1557, 1978. 